\title{
Prime-Boost Vaccination Using Chemokine-Fused gp120 DNA and HIV Envelope Peptides Activates Both Immediate and Long-Term Memory Cellular Responses in Rhesus Macaques
}

\author{
Hong Qin, ${ }^{1,2}$ Pramod N. Nehete, ${ }^{3}$ Hong He, ${ }^{2,4}$ Bharti Nehete, ${ }^{3}$ Stephanie Buchl, ${ }^{3}$ \\ Soung-chul Cha, ${ }^{1,2}$ Jagannadha K. Sastry, ${ }^{2,3,4}$ and Larry W. Kwak ${ }^{1,2}$ \\ ${ }^{1}$ Department of Lymphoma and Myeloma, M. D. Anderson Cancer Center, The University of Texas, Houston, TX 77030, USA \\ ${ }^{2}$ Center for Cancer Immunology Research, M. D. Anderson Cancer Center, The University of Texas, Houston, TX 77030, USA \\ ${ }^{3}$ Department of Veterinary Sciences, M. D. Anderson Cancer Center, The University of Texas, Bastrop, TX 78602, USA \\ ${ }^{4}$ Department of Immunology, M. D. Anderson Cancer Center, The University of Texas, Houston, TX 77030, USA
}

Correspondence should be addressed to Jagannadha K. Sastry, jsastry@mdanderson.org and

Larry W. Kwak, 1kwak@mdanderson.org

Received 21 November 2009; Accepted 2 March 2010

Academic Editor: Hanchun Yang

Copyright () 2010 Hong Qin et al. This is an open access article distributed under the Creative Commons Attribution License, which permits unrestricted use, distribution, and reproduction in any medium, provided the original work is properly cited.

\begin{abstract}
HIV vaccine candidates with improved immunogenicity and induction of mucosal T-cell immunity are needed. A prime-boost strategy using a novel HIV glycoprotein 120 DNA vaccine was employed to immunize rhesus macaques. The DNA vaccine encoded a chimeric gp120 protein in fusion with monocyte chemoattractant protein-3, which was hypothesized to improve the ability of antigen-presenting cells to capture viral antigen through chemokine receptor-mediated endocytosis. DNA vaccination induced virus-reactive T cells in peripheral blood, detectable by T cell proliferation, INF $\gamma$ ELISPOT and sustained IL-6 production, without humoral responses. With a peptide-cocktail vaccine containing a set of conserved polypeptides of HIV-1 envelope protein, given by nasogastric administration, primed T-cell immunity was significantly boosted. Surprisingly, long-term and peptide-specific mucosal memory T-cell immunity was detected in both vaccinated macaques after one year. Therefore, data from this investigation offer proof-of-principle for potential effectiveness of the prime-boost strategy with a chemokine-fused gp120 DNA and warrant further testing in the nonhuman primate models for developing as a potential HIV vaccine candidate in humans.
\end{abstract}

\section{Introduction}

Acquired immunodeficiency syndrome (AIDS) is a lifethreatening viral disease caused by a severely impaired immune defense mechanism resulting from devastation of $\mathrm{CD}^{+} \mathrm{T}$ lymphocytes by human immunodeficiency virus1 (HIV-1). A fundamental concept in controlling AIDS development is to prevent the loss of $\mathrm{CD} 4^{+} \mathrm{T}$ cells, which may be achieved by inhibiting intracellular viral replication and eliminating virus-infected cells. A therapeutic HIV vaccine that aims at activating adaptive $\mathrm{T}$-cell immunity to prevent intercellular spread of viruses is therefore required to curb the rapid worldwide spread of this disease. Although enormous efforts have been made to test a variety of vaccine strategies, the generation of an HIV vaccine with potent antiviral effects remains elusive. One major obstacle hampering vaccine development is the low magnitude of vaccine-elicited immunity. To overcome this issue, current attention is mainly focused on developing strategies to enhance the immunogenicity of HIV vaccine formulations and the induction of mucosal immunity. These attempts have included the delivery of HIV antigen genes by live recombinant adenovirus or mycobacterium, resulting in an improved HIV-specific immune response $[1,2]$. The critical role of dendritic cells in initiating the adaptive immune response is well recognized by their function in capturing, processing, and presenting antigens. Other strategies that specifically target delivery of antigens to dendritic cells may therefore increase the potency of antigen-specific immune responses [3-5]. Chemokines are a family of chemotactic polypeptides 
that facilitate the homing of antigen-bearing dendritic cells to draining lymph nodes. Chemokine-induced signal transduction is triggered by the binding of chemokines with Gprotein-coupled receptors and is subsequently terminated by the clathrin-mediated endocytosis of the chemokinereceptor complex. Given these features of chemokine receptors on dendritic cells, we previously described achievement of triggering potent T-cell responses by fusion of candidate, otherwise non-immunogenic antigens with chemokines, hypothesizing that the chemokine motif in the chimeric proteins would direct antigens to dendritic cells or other antigen-presenting cells in the body. Specifically, using HIV189.6 envelope glycoprotein 120 as a model antigen, we verified that genetic fusion of this candidate HIV antigen with chemokines dramatically increased its immunogenicity in mice [6]. Both systemic and mucosal HIV-specific Tcell immunities were elicited upon immunizing mice with plasmid DNA encoding the full-length gp120 gene fused with monocyte chemotactic protein-3 (MCP3). In the present proof-of-principle pilot study, we show for the first time that an MCP3-fused gp120 DNA vaccine elicited virus-specific Tcell immunity in rhesus macaques at a dose of only $20 \mu \mathrm{g}$ of plasmid DNA. The elicited T-cell response was boosted by nasal administration of a peptide-cocktail consisting of six highly conserved epitopes of the HIV-1 envelope protein. A highly desirable feature of this prime-boost strategy was the induction of long-term mucosal T-cell immunity.

\section{Materials and Methods}

2.1. Vaccination of Animals. The macaque study was performed under a protocol approved by the Institutional Animal Care and Use Committee of The University of Texas, M. D. Anderson Cancer Center. Two male rhesus macaques of Indian origin (designated J8 and J160) from the institution's specific pathogen-free breeding colony at the Science Park campus in Bastrop, Texas were used for the study. The animal facility is fully accredited by the Association for Assessment and Accreditation of Laboratory Animal Care International. The plasmid DNA encoding MCP3-fused gp120 was generated as described in our previous studies [6]. The DNA was precipitated in the presence of $1 \mu \mathrm{m}$ gold particles (BioRad, Hercules, CA) and the polycation spermide by the addition of calcium chloride. The DNA-attached gold particles were washed and resuspend in ethanol, coated onto the inner wall of Gold-Coat tubing (BioRad), dried and cut into 0.5" length cartridge. These cartridges were inserted into the cartridge holder of the Helio Gene Gun (BioRad). By helium discharge the DNA was directly delivered into skin. The particle-coated plasmid DNA was delivered intradermally using helium gene gun (BioRad) at 400 psi. A total amount of approximate $20 \mu \mathrm{g}$ DNA was evenly distributed at shaved areas on each arm or leg. The booster dose of the peptide-cocktail vaccine consists of six highly conserved HIV-1 envelope peptides (each at $100 \mu \mathrm{g} /$ dose/macaque). The peptide synthesis, amino acid sequence, and immunogenicity were reported previously [7-9]. The peptide-cocktail was administered by nasogastric route along with a mutant cholera toxin, CT2*
( $10 \mu \mathrm{g} / \mathrm{dose} / \mathrm{monkey)}$ that we reported previously to be a safe and effective mucosal adjuvant in murine and primate models $[10,11]$ in $600 \mu \mathrm{L}$ sterile PBS.

2.2. Collection of Samples. Peripheral venous blood samples collected in sodium heparin at different time points were centrifuged to separate plasma and buffy coat. Peripheral blood mononuclear cells (PBMC) were separated from buffy coat by centrifugation through Ficoll-Paque density gradients and were used for various immune assays. Also, serum samples were collected and stored at $-80^{\circ} \mathrm{C}$ for analysis of the neutralizing antibody. The intraepithelial lymphocytes (IEL) from the colon biopsy sections of the macaques were isolated by using modifications of previously described procedures [12]. Briefly, 10-15 samples of colon biopsy ( $2 \mathrm{~mm}$ each) were collected and rinsed in cold PBS. The tissue was treated with dithiothreitol (DTT, $1.5 \mathrm{mg} / \mathrm{mL}$ ) in Hank's balanced salt solution (HBSS) for 30 minute at RT with agitation to remove mucus. The tissue were then treated with $0.75 \mathrm{mM}$ EDTA in $\mathrm{Ca}^{2+}$ - and $\mathrm{Mg}^{2+}$-free HBSS at RT for 60 minute with stirring. After the treatment, the supernatants containing epithelial cells were removed and digested with $15 \mathrm{U}$ of collagenase D and DNAse I in complete RPMI 1640 medium containing $100 \mathrm{U}$ of penicillin and $100 \mathrm{U}$ of streptomycin per $\mathrm{ml}, 5 \mathrm{ml} \mathrm{L}$-glutamine, $5 \mathrm{ml}$ HEPES buffer, and $10 \% \mathrm{FCS}$ at $37^{\circ} \mathrm{C}$ with rapid shaking for 2 hours. The resulting cell suspensions were passed through stainless steel screen cups to remove the residual tissue fragments. The cells were washed and resuspended in complete RPMI 1640 medium and enriched for lymphocytes by isotonic discontinuous Percoll (Sigma) density gradients (35 and 60\% [vol/vol]) at $1,000 \mathrm{~g}$ for 20 minute at $4^{\circ} \mathrm{C}$. The lymphocyte band at the interface between the 35 and $60 \%$ Percoll layers was collected. Viability of lymphocytes was greater than $95 \%$ as determined by the Trypan blue dye exclusion method. Aliquots of freshly-isolated cells were stained with fluorescently-labeled antibodies for flow cytometry analysis.

2.3. Proliferation Assay. Proliferative responses of PBMC isolated from immunized macaques were determined using the standard $\left[{ }^{3} \mathrm{H}\right]$ thymidine incorporation assay as described earlier [13]. Briefly, $100 \mu \mathrm{L}$ aliquots of the cell suspensions $\left(1 \times 10^{5}\right.$ cells in complete RPMI) were dispersed into triplicate wells of a round-bottom 96-well microtiter plate and incubated with the HIV-1 envelope peptides $(10 \mu \mathrm{g} / \mathrm{mL}$ final concentration) as a single mixture (designated as pepmix) and the cell-free heat-inactivated $\mathrm{SHIV}_{89.6 \mathrm{P}}$ (equivalent to $50 \mathrm{ng} / \mathrm{ml}$ final concentration of p27) prepared by incubating for 60 minute at $56^{\circ} \mathrm{C}$. The proliferative response was calculated as the fold increase in radioactivity with the test antigen over that for cells cultured in the medium alone and is represented as the stimulation index (SI). The responses to peptides were considered positive when the SI values are 2.0 or greater, and also 2.0 above the value for the negative control peptide (from human papilloma virus at $10 \mathrm{mg} / \mathrm{mL}$ final concentration) or medium control used in the same experiment. No differences were observed between the negative control peptide and culture medium as control treatments. 
2.4. Measurement of IFN- $\gamma$ Production by ELISPOT Assay. PBMC isolated from immunized macaques were stimulated with the mixture of the HIV-1 envelope peptides and cell-free, heat-inactivated $\mathrm{SHIV}_{89.6 \mathrm{P}}$ for determining the antigen-specific interferon- $\gamma$ (IFN- $\gamma$ )-producing cells by the ELISPOT analyses, as reported earlier [13]. The membranes were read by an independent agency (Zellnet Consulting Inc., New York, NY, USA) using the KS-ELISPOT automatic system (Carl Zeiss, Inc., Thornwood, NY, USA) for quantitative analyses of the number of IFN- $\gamma$ spot forming cells (SFC) for $10^{5}$ input PBMC. Responses were considered positive, according to criteria described in the literature [13], which is a minimum of five SFC per well and at least double the number obtained in cells cultured in the medium.

2.5. Cytokine Analysis by Cytokine Bead Array (CBA). Single cell suspensions of PBMC from the immunized monkeys were cultured at a density of $1 \times 10^{5}$ per $\mathrm{mL}$ with or without the HIV-1 envelope peptide mixture and cell-free heatinactivated SHIV $_{89.6 P}$ to detect antigen-specific cytokine production. Culture supernatants were collected $36 \mathrm{~h}$ after incubation, and the level of cytokines (IL-2, IL-4, IL-6, IL-10, IL-12p70, IFN- $\gamma$, and TNF- $\alpha$ ) was determined by cytometric bead array (CBA) kit (Lincoplex multiple cytokine assay, Millipore), according to manufacturer's instructions. Antigenspecific cytokine production was measured as the cytokine concentration in culture supernatants of antigen-stimulated cells subtracting that without antigen.

2.6. Intracellular Cytokine Analyses. The PBMC along with lamina propria lymphocytes (LPL) isolated from the colon biopsies as previously described [13], were used for the analyses of antigen-specific intracellular cytokine production using the following antihuman monoclonal antibodies that cross-react with rhesus macaques: CD3 (clone SP34-2, PECy7-labeled), CD8a (clone RPA-T8, Alexa 700-labeled), CD28 (clone L293, PerCP-Cy5.5-labeled), CD95 (clone DX2, APC-labeled), and IFN- $\gamma$ (clone B27, FITC-labeled; all from BD Biosciences, San Jose, Calif, USA), CD4 (clone OKT4, Pacific blue-labeled; eBioScience, San Diego, Calif, USA), and Aqua (live/dead staining kit) from Invitrogen (Carlsbad, Calif, USA) to detect dead cells. The $\mathrm{CD} 4^{+}$and $\mathrm{CD}^{+} \mathrm{T}$ cells that produce IFN- $\gamma$ in response to stimulation with various reagents were analyzed by the standard intracellular cytokine assay procedure, as previously described [14]. Samples were collected on a three-laser CYAN ADP instrument (Dako Cytomation, Glostrup, Denmark) and analyzed using FloJo software (Tree Star, Ashland, OR). For data acquiring and analyzing, $\mathrm{CD}^{+} \mathrm{CD}^{+}$or $\mathrm{CD}^{+}{ }^{+} \mathrm{CD} 4^{+} \mathrm{T}$ cells initially gated from live lymphocytes were further gated for CD95 memory phenotype. The gated $\mathrm{CD} 4^{+}$or $\mathrm{CD} 8^{+}$memory $\mathrm{T}$ cells were then analyzed for positive staining of IFN $\gamma$.

\section{Results and Discussion}

We used nonhuman primates to evaluate the translational potential for the chemokine-fusion strategy in the development of a novel HIV vaccine. Two macaques immunized with the plasmid DNA encoding MCP3-gp120 fusions demonstrated virus-reactive T-cell immunity at week 12, evidenced by the proliferation of T cells (Figure 1(a)) and IFN- $\gamma$ producing cells in response to in vitro stimulation with heat-inactivated SHIV $_{89.6 P}$ (Figure 1(b)). Peptidecocktail vaccine boosted the viral-reactive T-cell immunity, as evidenced by the significant elevation of IFN- $\gamma$ producing cells in response to the heat-inactivated $\mathrm{SHIV}_{89.6 \mathrm{P}}$ in one of the two monkeys (Figure 1(b) \#J160). The peptidecocktail-specific response was readily detected after peptide boost, confirming the immunogenicity of the peptidecocktail vaccine (Figure 1(c)). The macaque (\#J8) that was weakly responsive to the peptide boost (Figure 1(b) \#J8) also showed a low magnitude of peptide-cocktail-specific systemic cellular responses (Figure 1(c)). PBMC cultured with medium or treated with irrelevant human papilloma virus peptide failed to show positive responses (data not shown). We were unable to detect antibody responses, either by neutralization assay or by Western blot (data not shown).

To further investigate changes in the DNA vaccineinduced microenvironment that may facilitate the development of memory immunity, we monitored virus-reactive, PBMC cytokine induction throughout the entire primeboost schedule. Strikingly, among a panel of cytokines including IL-2, IL-4, IL-6, IL-10, IL-12p70, IFN- $\gamma$, and TNF- $\alpha$, production of IL- 6 was found to be significantly increased upon initial vaccination with MCP-3-fused gp120 plasmid DNA. Of interest, immune cells harvested from the monkey \#J160 during the boost period (W32-39) were considerably more active in producing IL-6, compared with PBMC collected from the macaque \#J8 (Figure 1(d)).

We analyzed colon biopsy samples collected from the macaques one year after the final peptide-cocktail boost. During this year-long rest period the two vaccinated macaques received no further treatment. Surprisingly, antigen-specific CD4 and CD8 memory T cells were detected in lamina propria lymphocytes (LPL) isolated from the colon, suggesting long-term mucosal immunity. Specifically, after in vitro stimulation with the peptide-mix, subsets of IFN- $\gamma$-producing $\mathrm{CD}^{+} \mathrm{CD}^{+} \mathrm{CD}^{-} 5^{+}$and $\mathrm{CD} 3^{+} \mathrm{CD} 8^{+} \mathrm{CD} 95^{+}$ $\mathrm{T}$ cells were readily detected in both vaccinated macaques (Figure 2). The memory $\mathrm{T}$ cell response was found only in LPL samples, but not in PBMC (Figure 2). These results suggest that the immunization regimen induced mucosal memory cellular immune responses, which likely resulted from directly delivering viral antigen to the gastrointestinal mucosa by nasogastric administration of the peptidecocktail.

Growing evidence has emphasized the central role of cellular immunity in the control of HIV infection. For example, the prevalence of virus-specific cytotoxic $\mathrm{T}$ cells has been associated with reduced viral RNA load and delayed clinical progression of the disease [15]. In nonhuman primate models, depletion of $\mathrm{CD}^{+} \mathrm{T}$ lymphocytes resulted in marked viral replication in chronically infected animals [16]. It is thus important to focus development of HIV vaccines that generate cellular immunity for therapeutic benefit. In this pilot study, we observed that the MCP3fused gp120 DNA vaccine elicited the viral-reactive T-cell immunity in rhesus macaques that were vaccinated with 


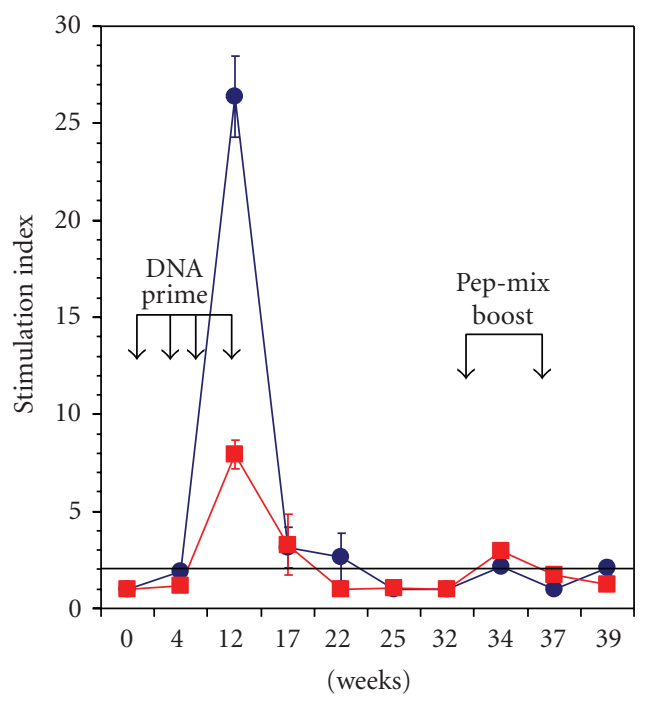

Virus-reactive proliferation

$\rightarrow$ J8

$-\mathrm{J} 160$

(a)

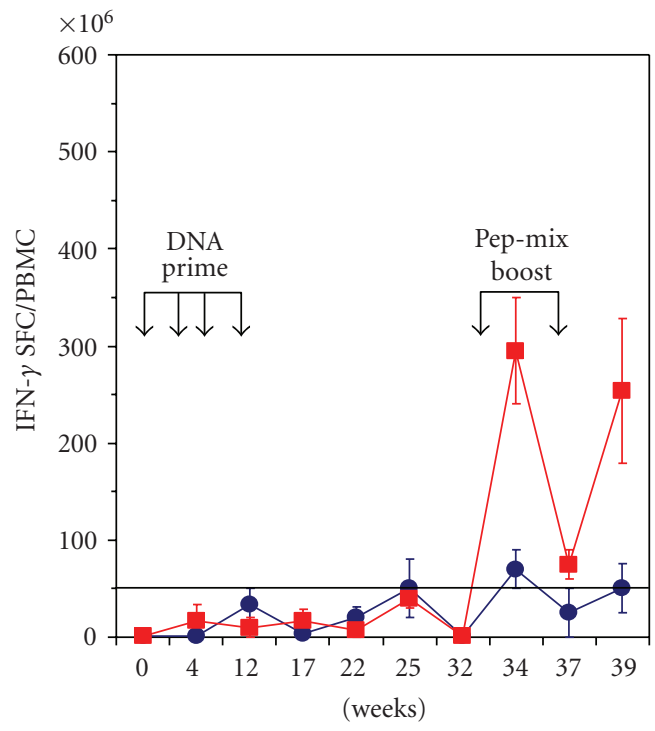

Pep-mix-specific IFN- $\gamma$ producing cells

$-\mathrm{J} 8$

J160

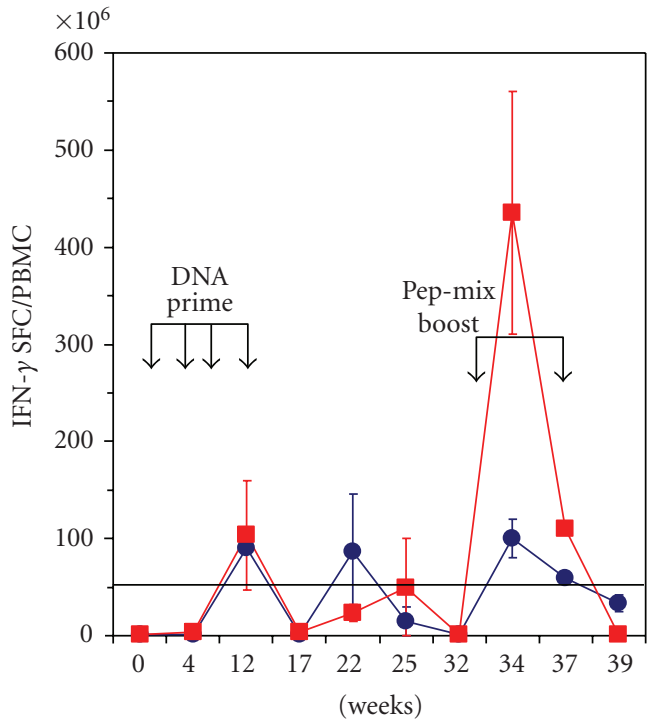

Virus-reactive IFN- $\gamma$ producing cells

- J8

$-\mathrm{T} 160$

(b)

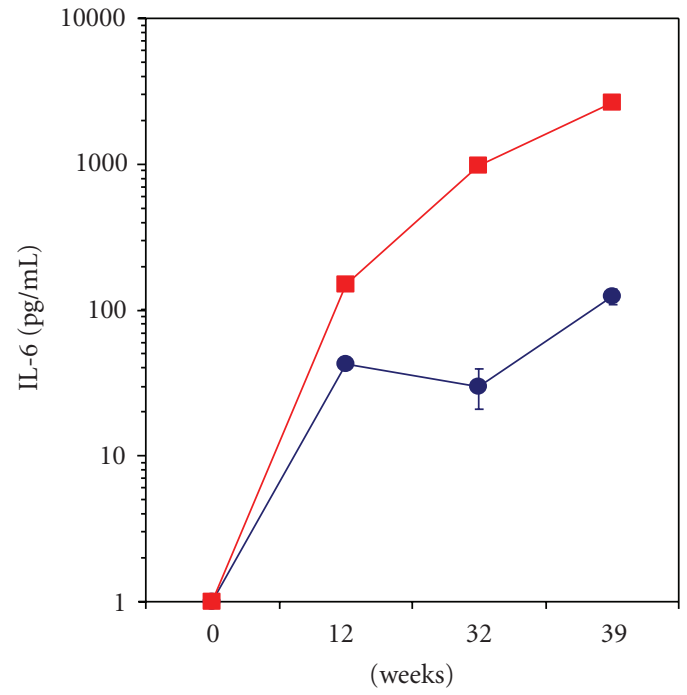

Virus-reactive cytokine production

$\rightarrow$ J8

$\mathrm{J} 160$

(c)

(d)

FIGURE 1: Immunization of rhesus macaques with plasmid DNA encoding MCP3-gp120 fusion primed viral-reactive T-cell immunity, which was further boosted by HIV-1 envelope peptide-cocktail vaccine. The DNA priming schedule included four rounds of immunization at 1month intervals. Two rhesus macaques were immunized with $20 \mu \mathrm{g}$ of the plasmid DNA by gene gun injection at weeks $0,4,8$, and 12 . The boosting schedule included a peptide-cocktail vaccine that contains six highly conserved peptides from the HIV-1 envelope protein. After a rest period of 20 weeks following the final round of DNA vaccination to allow for the establishment of memory T cells, the peptide-cocktail boosts were administered by intranasal route along with a mutant cholera toxin on Weeks 32 and 37. Peripheral blood mononuclear cells collected after DNA vaccination revealed viral-reactive T-cell immunity as evidenced by the cell proliferation (a) and IFN- $\gamma$ producing cells (b) in response to in vitro stimulation with cell-free, heat-inactivated SHIV $_{89.6 \mathrm{P}}$ antigen-specific T-cell immunity was boosted by the peptidecocktail vaccine, especially in \#J160 showing significant elevation of IFN- $\gamma$ producing cells in response to heat-inactivated SHIV $89.6 \mathrm{P}$ ) (b). The immunogenicity of the peptide vaccine was confirmed by analysis of peptide-specific T-cell immunity (c). Vaccine-induced cytokine production was examined using LINCOplex multiple cytokine luminescent assay. Among the cytokines assayed, IL- 6 was predominantly detected in both macaques after DNA vaccination at Week 12. Virus-reactive IL-6 secretion was more apparent in monkey \#J160, particularly during the period of peptide-cocktail boost. 


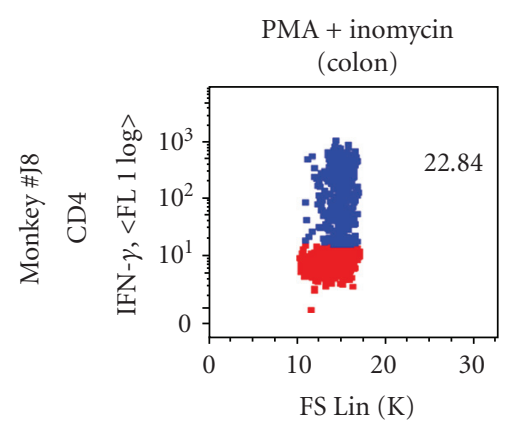

(a)

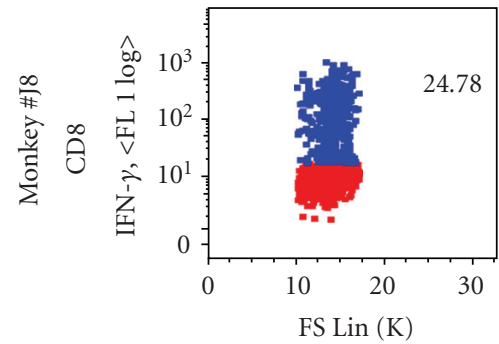

(e)

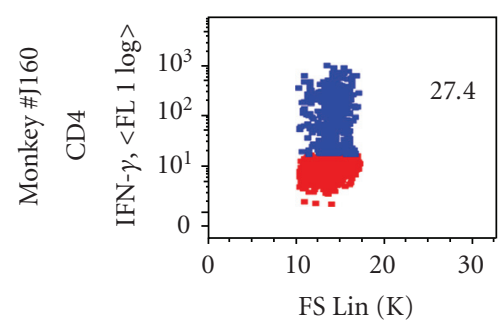

(i)

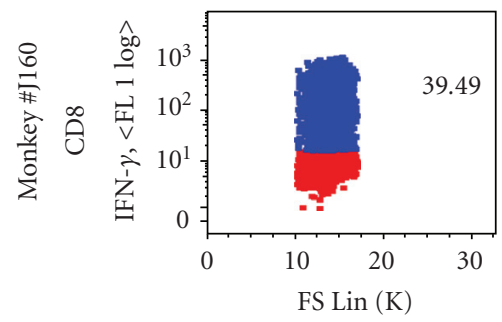

(m)

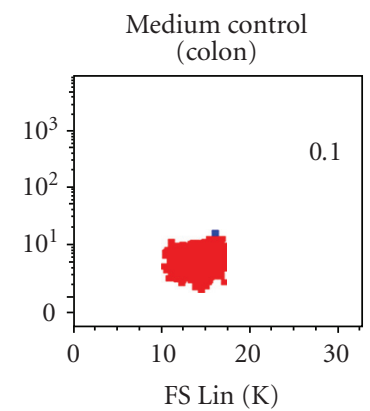

(b)

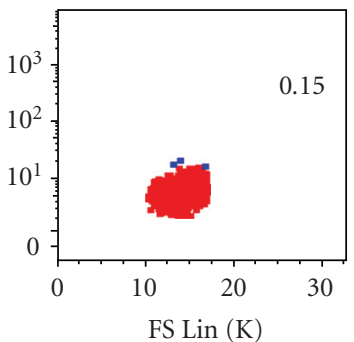

(f)

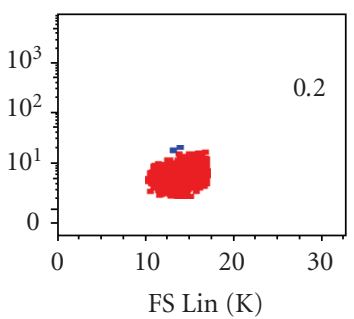

(j)

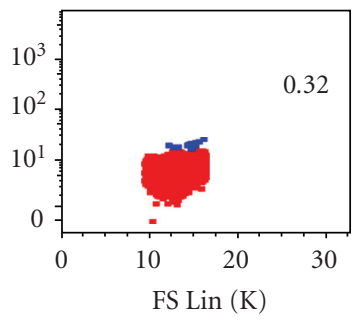

(n)

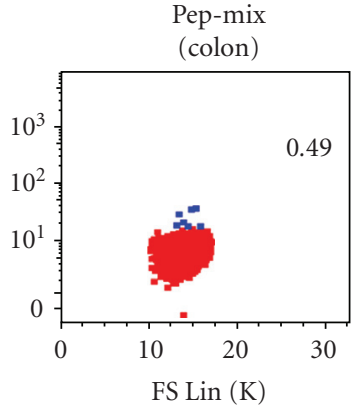

(c)

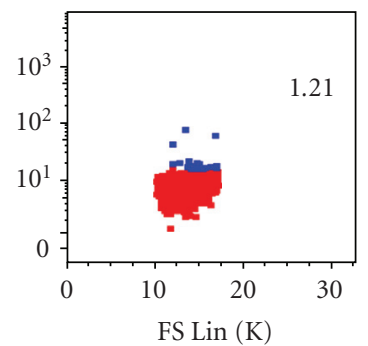

(g)

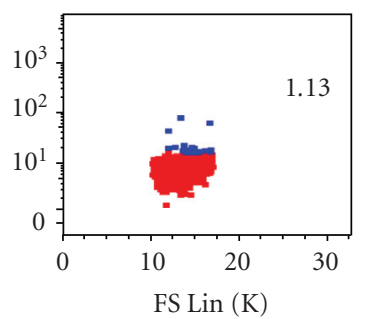

(k)

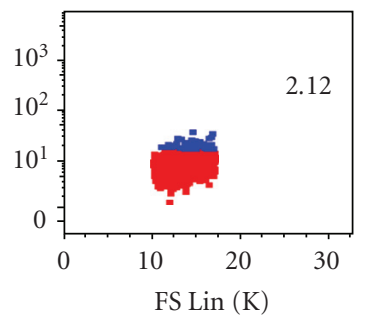

(o)

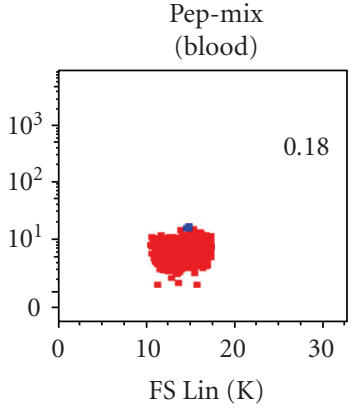

(d)

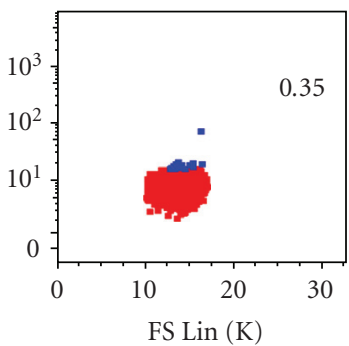

(h)

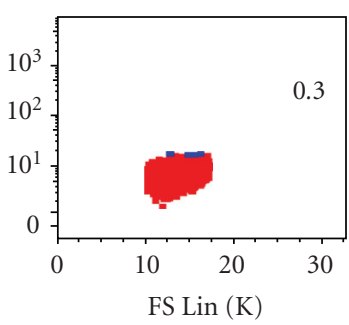

(1)

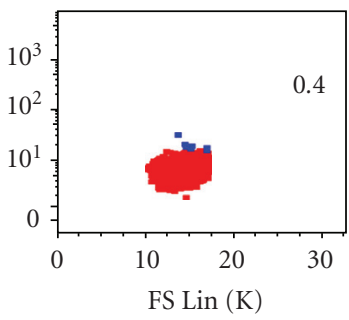

(p)

FIgURE 2: The immunization strategy elicited mucosal long term memory T-cell immune responses. Production of IFN- $\gamma$ by $\mathrm{CD} 3^{+} \mathrm{CD} 4^{+}$ or $\mathrm{CD}^{+} \mathrm{CD}^{+}$memory $\mathrm{T}$ cells isolated from colon was analyzed in the vaccinated macaques one year after final peptide-cocktail boost. Lamina propria lymphocytes (LPL) from colon biopsy samples were stimulated with peptide-mix or mitogens for $6 \mathrm{~h}$. Both untreated (control) and stimulated cells were stained for surface markers, followed by fixation, permeabilization, and intracellular staining of IFN- $\gamma$. Live cells were identified by gating on Aqua-negative cells. The cells gated on $\mathrm{CD} 3{ }^{+} \mathrm{CD} 4^{+}$and $\mathrm{CD} 3{ }^{+} \mathrm{CD} 8^{+}$were further separated as memory population according to the expression of CD95 (data not shown). The percentage values indicate the population of IFN- $\gamma-$ producing $\mathrm{CD}^{+} \mathrm{CD}^{+} 5^{+} \mathrm{CD} 4^{+}$or $\mathrm{CD} 3^{+} \mathrm{CD} 95^{+} \mathrm{CD} 8^{+}$lymphocytes.

as little as $20 \mu \mathrm{g}$ of plasmid DNA by gene gun injection. Although the individual response in terms of immunity potency was not robust, the immunogenicity of MCP3gp120 fusions DNA vaccine in a nonhuman primate model was supported from the data obtained. Thus, future studies are warranted to confirm and extend the data from the proof-of-principle study employing bigger cohort of animals combined with determining protective efficacy against viral challenge. Furthermore, optimizing DNA delivery may also help to enhance the immunogenicity of the vaccine strategy. For example, we currently developed an immune potentiating strategy by inducing a sterile inflammation at vaccination sites, which significantly potentiated DNA vaccine-induced adaptive immunity [17]. Nevertheless, the 
take-home message that MCP3-gp120 DNA vaccine induced $\mathrm{T}$-cell immunity in macaques provides us with a rationale to further optimize the chemokine-fusion strategy for designing the next generation of HIV vaccines.

In contrast to activation of T-cell immunity, the fusion vaccine failed to induce antibody response. This may imply that antigen cross-presentation mechanism was predominantly responsible for the observed adaptive immunity. A striking feature of the chemokine fusion strategy is its receptor-mediated endocytosis that facilitates loading antigens to the MHC Class I pathway and thus polarizes Th1 response [18]. Accordingly, we also observed in mice that chemokine-chicken ovalbumin fusion vaccine stimulated expansion of adoptively transferred antigen-specific $\mathrm{CD}^{+}$ (OT-I), but not CD4 ${ }^{+}$(OT-II) T cells (unpublished data).

Mucosal entry is a major route of HIV infection. A recent clinical study suggests that blocking mucosal viral transmission would be critical to the prevention of systemic spread of HIV [19]. The local mucosal immunity brings an additional beneficial influence to disease control. Therefore, an ideal HIV vaccine should be designed to activate both systemic and local immune responses. Our current study showed that nasogastric administration of multivalent antigen peptides not only boosted the genetic fusion-primed $\mathrm{T}$ cell immunity, but also elicited mucosal immunity. The antigen-specific, long-lasting $\mathrm{T}$ cell immunity was readily detected a year later after final vaccination, which renders our prime-boost strategy with an extra advantage in the aspect of prophylactic value.

Cytokines produced in the microenvironment may be mechanistically associated with the elicited immune response. Of particular interest in the current study was the significant increase in IL-6 upon vaccination with chemokine-fused gp120 plasmid DNA. This T-cell-derived cytokine was initially identified as a B-cell growth and differentiation factor [20] and was subsequently discovered to have a variety of biological activities, such as stimulating differentiation of myeloid precursors, activating $\mathrm{T}$ cells by enhancing IL-2 responsiveness, and regulating acute phase reaction [21-23]. The stimulatory function of IL- 6 on diverse immune cells renders this cytokine a potential immune adjuvant in the development of adaptive immune response. The current study does not allow us to differentiate the mechanism that caused the production of IL-6. Since IL6 is not constitutively produced by $\mathrm{T}$ cells, rather it is the consequence of tissue damage as seen in the case of viral or bacterial infection, we speculate that the viral gp120 protein component in the fusion construct of the DNA vaccine might be the stimulator for IL-6 production. Regardless of the underlining mechanism, our observation that sustained IL6 production was correlated with vaccine boosting suggests a potential role of this cytokine in the maintenance of longterm memory immunity.

Future studies exploring the use of the chemokine-fusion strategy to develop vaccines targeting other HIV antigens including pol and gag gene products may be worthwhile. It has been reported that multivalent vaccines demonstrated superior antiviral effect over single antigen-derived vaccines [24]. Combined with more efficient vaccinia viral vectors
[24], we expect that DNA vaccines encoding multivalent HIV antigens fused with chemokines would be promising candidates for HIV vaccines.

\section{Conflict-of-Interest Disclosure}

The authors declare no competing financial interests.

\section{Acknowledgments}

H. Qin and P. N. Nehete equally contributed to the work. We thank Dr. D. Montefiori (Duke University Medical Center, Durham, NC) for the support in performing neutralizing antibody studies, and Dr. Ashok Chopra (The University of Texas Medical Branch, Galveston, TX) for providing the purified preparation of the mutant cholera toxin, CT2*. This work was supported by a translational research grant from Leukemia and Lymphoma Society (LWK), RO1 (AI 426969) and R21(AI 065402) grants from NIAID (KJS).

\section{References}

[1] A. T. Catanzaro, R. A. Koup, M. Roederer, et al., "Phase 1 safety and immunogenicity evaluation of a multiclade HIV-1 candidate vaccine delivered by a replication-defective recombinant adenovirus vector," Journal of Infectious Diseases, vol. 194, no. 12, pp. 1638-1649, 2006.

[2] M. J. Cayabyab, A.-H. Hovav, T. Hsu, et al., "Generation of $\mathrm{CD}^{+} \mathrm{T}$-cell responses by a recombinant nonpathogenic Mycobacterium smegmatis vaccine vector expressing human immunodeficiency virus type 1 Env," Journal of Virology, vol. 80, no. 4, pp. 1645-1652, 2006.

[3] A. Biragyn, K. Tani, M. C. Grimm, S. Weeks, and L. W. Kwak, "Genetic fusion of chemokines to a self tumor antigen induces protective, T-cell dependent antitumor immunity," Nature Biotechnology, vol. 17, no. 3, pp. 253-258, 1999.

[4] A. Biragyn, P. A. Ruffini, C. A. Leifer, et al., "Toll-like receptor 4 -dependent activation of dendritic cells by $\beta$-defensin 2 ," Science, vol. 298, no. 5595, pp. 1025-1029, 2002.

[5] A. Biragyn, M. Surenhu, D. Yang, et al., "Mediators of innate immunity that target immature, but not mature, dendritic cells induce antitumor immunity when genetically fused with nonimmunogenic tumor antigens," Journal of Immunology, vol. 167, no. 11, pp. 6644-6653, 2001.

[6] A. Biragyn, I. M. Belyakov, Y.-H. Chow, D. S. Dimitrov, J. A. Berzofsky, and L. W. Kwak, "DNA vaccines encoding human immunodeficiency virus-1 glycoprotein 120 fusions with proinflammatory chemoattractants induce systemic and mucosal immune responses," Blood, vol. 100, no. 4, pp. 11531159, 2002.

[7] P. N. Nehete, S. J. Schapiro, P. C. Johnson, K. K. Murthy, W. C. Satterfield, and K. J. Sastry, "A synthetic peptide from the first conserved region in the envelope protein gp160 is a strong Tcell epitope in HIV-infected chimpanzees and humans," Viral Immunology, vol. 11, no. 3, pp. 147-158, 1998.

[8] P. N. Nehete, W. C. Satterfield, C. M. Matherne, R. B. Arlinghaus, and K. J. Sastry, "Induction of human immunodeficiency virus-specific T cell responses in rhesus monkeys by synthetic peptides from gp160," AIDS Research and Human Retroviruses, vol. 9, no. 3, pp. 235-240, 1993.

[9] P. N. Nehete, B. P. Nehete, P. Manuri, L. Hill, J. L. Palmer, and K. J. Sastry, "Protection by dendritic cells-based HIV synthetic 
peptide cocktail vaccine: preclinical studies in the SHIV-rhesus model," Vaccine, vol. 23, no. 17-18, pp. 2154-2159, 2005.

[10] D. Lomada, R. Gambhira, P. N. Nehete, et al., "A two-codon mutant of cholera toxin lacking ADP-ribosylating activity functions as an effective adjuvant for eliciting mucosal and systemic cellular immune responses to peptide antigens," Vaccine, vol. 23, no. 4, pp. 555-565, 2004.

[11] G. T. Mercier, P. N. Nehete, M. F. Passeri, et al., "Oral immunization of rhesus macaques with adenoviral HIV vaccines using enteric-coated capsules," Vaccine, vol. 25, no. 52, pp. 8687-8701, 2007.

[12] J. Baig, D. B. Levy, P. F. McKay, et al., "Elicitation of simian immunodeficiency virus-specific cytotoxic $\mathrm{T}$ lymphocytes in mucosal compartments of rhesus monkeys by systemic vaccination," Journal of Virology, vol. 76, no. 22, pp. 1148411490, 2002.

[13] R. S. Veazey, M. Rosenzweig, D. E. Shvetz, et al., "Characterization of gut-associated lymphoid tissue (GALT) of normal rhesus macaques," Clinical Immunology and Immunopathology, vol. 82, no. 3, pp. 230-242, 1997.

[14] M.-C. Gauduin, A. Kaur, S. Ahmad, T. Yilma, J. D. Lifson, and R. P. Johnson, "Optimization of intracellular cytokine staining for the quantitation of antigen-specific $\mathrm{CD}^{+} \mathrm{T}$ cell responses in rhesus macaques," Journal of Immunological Methods, vol. 288, no. 1-2, pp. 61-79, 2004.

[15] L. Musey, J. Hughes, T. Schacker, T. Shea, L. Corey, and M. J. Mcelrath, "Cytotoxic-T-cell responses, viral load, and disease progression in early human immunodeficiency virus type 1 infection," New England Journal of Medicine, vol. 337, no. 18, pp. 1267-1274, 1997.

[16] J. E. Schmitz, M. J. Kuroda, S. Santra, et al., "Control of viremia in simian immunodeficiency virus infection by $\mathrm{CD}^{+}$ lymphocytes," Science, vol. 283, no. 5403, pp. 857-860, 1999.

[17] H. Qin, S.C. Cha, S. S. Neelapu, et al., "Vaccine site inflammation potentiates idiotype DNA vaccine-induced therapeutic T cell-, and not B cell-, dependent antilymphoma immunity," Blood, vol. 114, no. 19, pp. 4142-4149, 2009.

[18] R. Schiavo, D. Baatar, P. Olkhanud, et al., "Chemokine receptor targeting efficiently directs antigens to MHC class I pathways and elicits antigen-specific CD8 ${ }^{+}$T-cell responses," Blood, vol. 107, no. 12, pp. 4597-4605, 2006.

[19] T. Hirbod and K. Broliden, "Mucosal immune responses in the genital tract of HIV-1-exposed uninfected women," Journal of Internal Medicine, vol. 262, no. 1, pp. 44-58, 2007.

[20] T. Kinashi, N. Harada, and E. Severinson, "Cloning of complementary DNA encoding T-cell replacing factor and identity with B-cell growth factor II," Nature, vol. 324, no. 6092, pp. 70-73, 1986.

[21] J. Gauldie, C. Richards, D. Harnish, P. Lansdorp, and H. Baumann, "Interferon beta 2/B-cell stimulatory factor type 2 shares identity with monocyte-derived hepatocytestimulating factor and regulates the major acute phase protein response in liver cells," Proceedings of the National Academy of Sciences of the United States of America, vol. 84, no. 20, pp. 7251-7255, 1987.

[22] T. Andus, T. Geiger, and T. Hirano, "Recombinant human $\mathrm{B}$ cell stimulatory factor 2 (BSF-2/IFN- $\beta 2$ ) regulates $\beta$ fibrinogen and albumin mRNA levels in Fao-9 cells," FEBS Letters, vol. 221, no. 1, pp. 18-22, 1987.

[23] Y. Shabo, J. Lotem, M. Rubinstein, et al., "The myeloid blood cell differentiation-inducing protein MGI-2A is interleukin6," Blood, vol. 72, no. 6, pp. 2070-2073, 1988.
[24] N. A. Doria-Rose, C. Ohlen, P. Polacino, et al., "Multigene DNA priming-boosting vaccines protect macaques from acute $\mathrm{CD}^{+}$-T-cell depletion after simian-human immunodeficiency virus SHIV89.6P mucosal challenge," Journal of Virology, vol. 77, no. 21, pp. 11563-11577, 2003. 


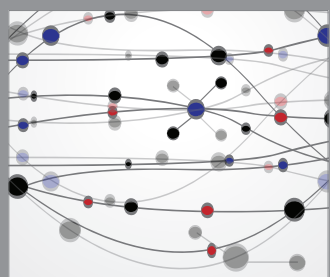

The Scientific World Journal
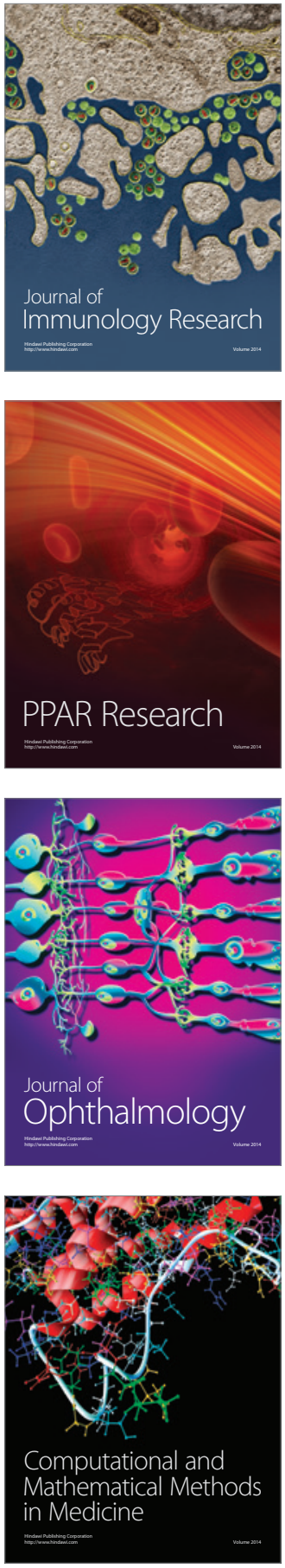

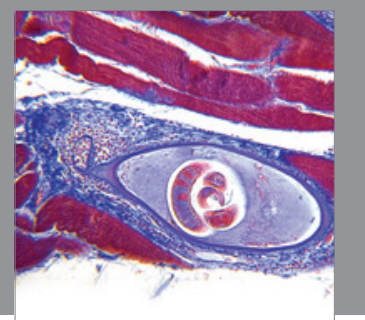

Gastroenterology

Research and Practice
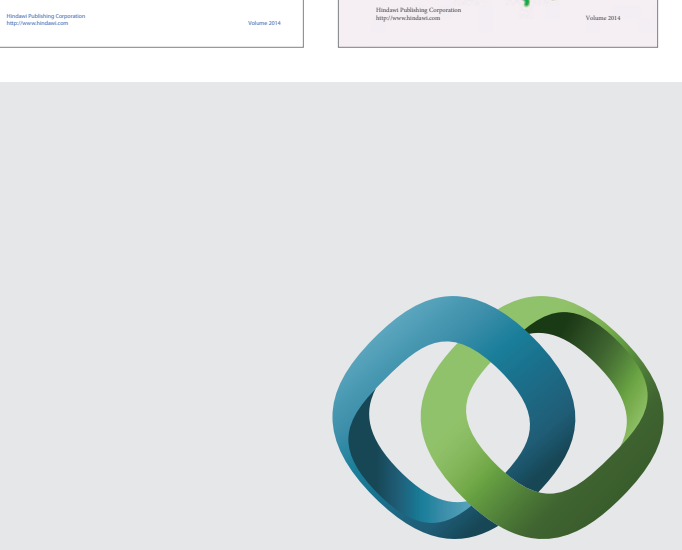

\section{Hindawi}

Submit your manuscripts at

http://www.hindawi.com
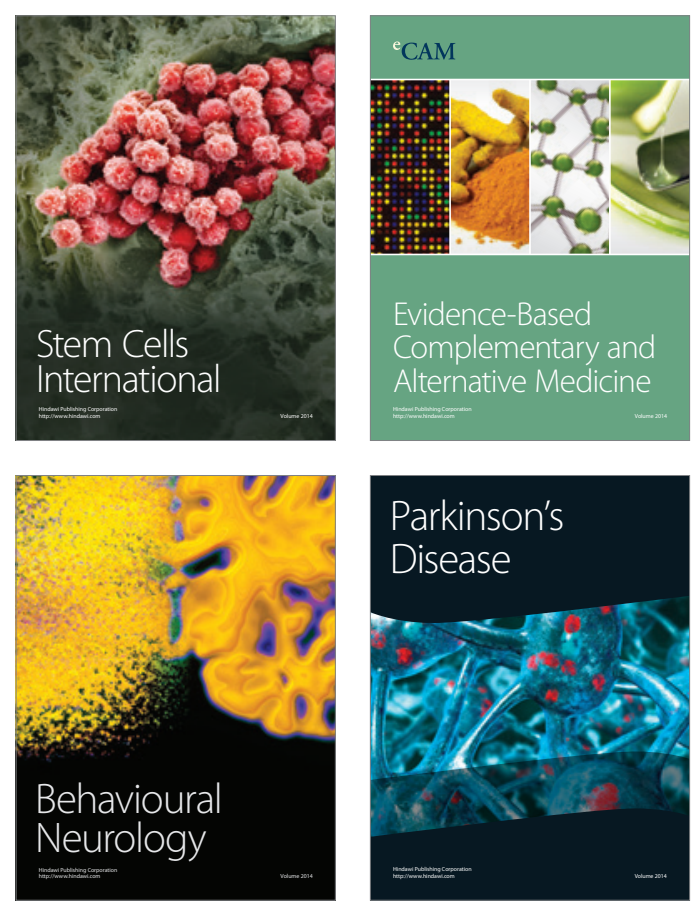

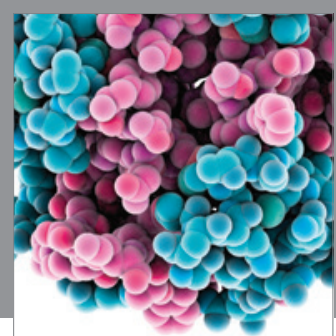

Journal of
Diabetes Research

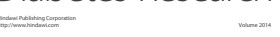

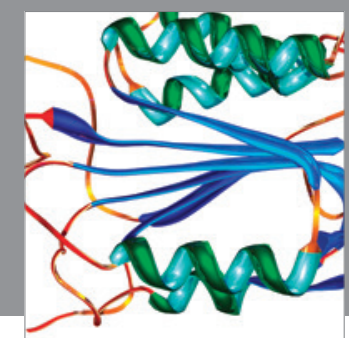

Disease Markers
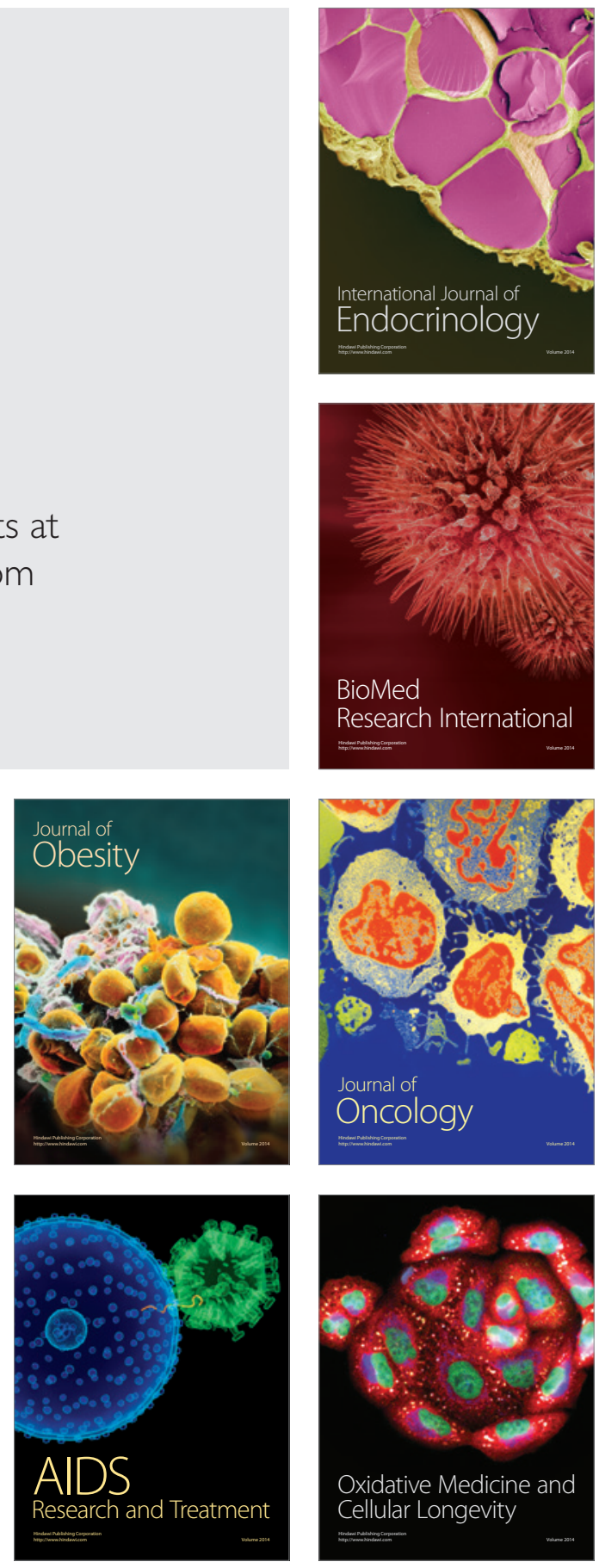